\title{
IMPLEMENTING FAST PART PROBING AND ERROR COMPENSATION ON MACHINE TOOLS
}

Kenneth W. Yee Robert J. Gavin

U.S. DEPARTMENT OF COMMERCE Natlonal Instltute of Standards and Tochnology Natlonal Engineering Laboratory Center for Manufacturing Engineering Automated Production Tochnology Division Galthersburg, MD 20899 



\section{IMPLEMENTING FAST PART PROBING AND ERROR COMPENSATION ON MACHINE TOOLS}

\section{Kenneth W. Yee Robert J. Gavin}

U.S. DEPARTMENT OF COMMERCE Natlonal instltute of Standards and Technology Natlonal Englneerlng Laboratory Center for Manufacturing EngIneering Automated Productlon Technology Division Galthersburg, MD 20899

October 1990

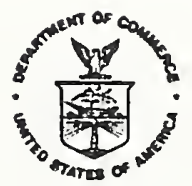

U.S. DEPARTMENT OF COMMERCE Robert A. Mosbacher, Secretary NATIONAL INSTTUTE OF STANDARDS AND TECHNOLOQY John W. Lyons, Director 

As a part of a Quality in Automation project, the Center for Manufacturing Engineering at the National Institute of Standards and Technology (formerly the National Bureau of Standards) has a need to implement fast part probing and real-time error compensation on both a computer-numericalcontrol $(\mathrm{CNC})$ machining center and a turning center. The purpose of this project is to control the machining process in order to minimize errors in the finished part. In small-batch (one to ten parts) manufacturing, traditional statistical quality-control or process-control techniques are not sufficient. One method of improving the accuracy of parts is to use process-intermittent gauging (i.e., part probing on the machine tool). The probing data may be used to adjust the depth of cut on the finish pass, or correct tool offsets to improve the next part. Fast part probing using a touch-trigger probe, with about one probe trip per second, is desired to minimize the time consumed by the measurement which reduces machining time and productivity. Probing cycles currently implemented by machine tool controllers use slow feed rates, about 5 inches per minute $(125 \mathrm{~mm} / \mathrm{min})$, and are slow in transferring the axes coordinates for the probed points to external computers usually through RS-232 distributed numerical control (DNC) interfaces. Consequently, they waste time.

In the near future we will also implement error compensation. The premise is that many machine tool errors are repeatable and predictable and that we can compensate for these systematic errors during the process, reducing the errors in the finished part. Such errors may be fixed geometric errors, such as lead-screw nonlinearity or straightness errors in the ways, or thermally induced errors such as spindle head growth [1]. Error compensation is intended to allow parts to be produced with errors approaching the repeatability limits of the machine tool even during much of the thermal warm-up period. This paper will discuss a method for implementing fast part probing and real-time error compensation on a wide variety of machine-tool controllers. The methodology for error compensation [2] and the mathematical model for the machine-tool errors [3] have been previously described.

We have inserted a black box between the position feedback elements of the axes of a machine tool and the machine-tool controller (MTC). This device independently and simultaneously counts the signals from the feedback element to produce the machine position and also includes an interface to send this position data to a PC-type computer which records the data at probe trip-points and will calculate the required error-compensation for each axis. The device allows fast part probing and realtime correction of positions of the machine-tool axes without intrusion into the MTC and in a way that is invisible to the machine tool. A minimum of hardware and software is MTC specific. For fast probing, the MTC merely executes a part program which moves the probe past the edge of the part with a feed rate of $100 \mathrm{in} / \mathrm{min}(2500 \mathrm{~mm} / \mathrm{min})$ and the device sends trip-point axes' positions to the PC. During inspection, the part location is well known (within a few thousandths of an inch [about a tenth of a millimeter]) since it has just been machined. The probing data is analyzed off-line by the PC after probing is completed. Part dimension correction can be implemented by updating tool offsets or part program values.

For error compensation, the signals from the position feedback device are modified to make the machine axes go to slightly different physical positions (as commanded by signals from the PC) than they would without the corrections applied. The PC has the axes-position data available and can compute a real-tin correction factor based on the positions along with temperature-sensor data which will be provided. The corrections will be calculated from the empirically determined model [3] for the current axes positions and temperatures. The coefficients for use in this model are determined from laser interferometer measurements of each machine-tool axis over the range of 
machine operating temperatures. A small number of selected temperatures that correlate with the thermally induced errors for the axes are used by the model. Generally, errors caused by specific machining conditions, e.g., tool deflection and servo lag, cannot be compensated. The device will work with a variety of MTCs with a minimum of modifications.

\section{Background}

Over the last five years, touch-trigger probes have become common on machine tools. The initial applications were primarily to reduce set-up time [4]. Recently, temperature control techniques and the use of glass scales have improved positioning of general-purpose machine tools. Advertised axis repeatabilities of one to three micrometers and accuracies about 3 to 5 times larger are the norm. On-machine inspection is now becoming more common. However, the desire to shave seconds from cycle times makes long inspection times unacceptable. In typical MTC canned probing cycles, the probe is fed towards the part slowly. The axes are rapidly braked to a stop at probe trip (or a programmed distance after trip). The MTC then must determine how to retract to the starting point since the stopping point is variable. In our system the MTC will always be in control, moving the probe quickly into and out of the part between programmed points--unaware of the probing process.

The Center for Manufacturing Engineering has previously implemented error compensation on several machine tools. Two of them are a $20 \mathrm{hp}(15 \mathrm{~kW})$ vertical machining center (VMC) and a $16.8 \mathrm{hp}(12.5 \mathrm{~kW})$ turning center (TC). The feedback elements for the VMC are linear glass scales, and the output signals are conditioned to appear as resolver signals (two sine wave signals that change in relative phase by 360 degrees for one rotation). The position information is obtained by piggybacking the counter-latches which contain the raw-count axes positions of the controller. This technique was possible only because the MTC manufacturer provided the physical location and logical address of the registers holding the position counts. The counts were simultaneously and independently latched and sent by a microcomputer to a minicomputer for off-line correction calculation. In these calculations, machine tool geometric errors as well as thermal growth of the spindle head were taken into account. The spindle growth was measured in both the $\mathrm{X}$ and $\mathrm{Y}$ directions by two linear variable differential transformers (LVDT) mounted at the end of the machine-tool table. Periodically, a tool was moved to these LVDT's where readings of displacement of the spindle head were taken. These displacement readings, along with the axes positions, were used to compute position corrections, which were used to produce an apparent phase shift between the sine waves making the controller stop the axis at a slightly different position than without the correction. The amount of control available allowed about \pm 3.5 milliinches $( \pm 0.1 \mathrm{~mm})$ change in the axis positions, which was invisible to the controller. The correction resolution was 30 microinches ( 0.7 micrometer). Only the $\mathrm{X}$ and $\mathrm{Y}$ axes of this machine were compensated since the primary application requiring the compensation was drilling, which is not sensitive to $\mathrm{Z}$-axis errors.

The turning center has a controller which facilitated the error compensation without any hardware modifications [5]. The machine has resolver feedback, but since the position correction is done entirely in software, the type of feedback is immaterial. An 8086 based single-board microcomputer, running at $8 \mathrm{MHz}$ with an 8087 math coprocessor, was used to calculate the corrections. Digital input/output (I/O) interface boards in the MTC provide axes positions to the microcomputer. The computed correction is fed back into the MTC by modifying the contents of the "following error" register which holds the difference between the programmed position and the 
position indicated from the processed resolver signals. The axis reaches the desired position when the following error is zero, and the modification of this following error causes the machine to stop at a different position than it would without the correction. The resolution of the correction is the resolution of the MTC. A new correction is calculated and inserted at every control cycle of 20 milliseconds. Both the $\mathrm{X}$ and $\mathrm{Z}$ axes of this machine have error compensation. Dimensional accuracy improvements on the order of 10 to 20 times, in diameter and length, were achieved over the cold-to-operating temperature range, and straightness was improved by a factor of 4 to 5 over a 2.7-in (70-mm) length.

The previous two cases are very MTC specific--one used hardware and the other software. The compensation systems used are only applicable to the specific MTC models.

\section{Approach}

The black box called the Real-Time Error Corrector (RTEC), with probing and compensating modes of operation, has been inserted between the position feedback devices and the MTC as shown in Figure 1. The RTEC independently processes the feedback signals and sends the positions (at trippoint for probing and continuously for compensation) to a PC/AT-compatible computer. This computer records the probe-trip axes positions and, for error compensation, will compute the required position corrections and send them back to the RTEC which will alter the position signals passed through to the MTC to implement the correction. In addition to resolvers and encoders, there are several types of position feedback devices in use today, all of which generate either resolver-type or encoder-type signals. Among them, the Inductosyn ${ }^{1}$ produces resolver-type signals while linear glass scales have signal conditioning available to produce either type of signal. Resolver-type and encoder-type signals are quite different and require different versions of the RTEC. Most modern machine tools use resolvers, encoders or linear glass scales, though the trend is more toward the use of encoders and glass scales. The signals from these devices will now be discussed.

Resolver-type signals, from resolvers or glass scales, have two sinusoidal waveforms, as shown in Figure 2. The electrical phase difference between the two waveforms is a function of angular position, changing $360^{\circ}$ as the resolver turns one full revolution [6]. The frequency is fixed by the excitation, typically about $2 \mathrm{kHz}$, and, with no axis motion, the resolver produces fixed signals such as shown in Figure 2a. If the axis moves in the positive direction by an amount that rotates the resolver 1/8th turn, the output signal will appear as in Figure $2 \mathrm{~b}$. Figure $2 \mathrm{c}$ illustrates the output signal when the axis then moves $3 / 8$ ths of a turn in the negative direction. To measure position, the RTEC must include circuitry to measure the relative phase between the reference and output signals and count the full cycles that pass by. For correction, a phase shifter would alter the phase of the output signal passed through to the MTC. The linear axis displacement for one rotation of the resolver is a function of the gear ratio between the resolver and the ball screw and the ball-screw

${ }^{1}$ Certain commercial equipment are identified in this paper in order to adequately specify the experimental procedure. Such identification does not imply recommendation or endorsement by the Hational Institute of Standards and Technology, nor does it imply that the equipment specified is necessarily the best for the purpose. 
pitch. The phase difference is generally measured with a resolution that corresponds to about 40 microinches (one micrometer) of axis movement. If the phase shifter increases the phase difference passed through to the MTC by this amount, the axis will stop 40 microinches sooner (less positive) than without the correction.

Encoder-type signals, shown in Figure 3, have two square-wave output signals (A and B) that are displaced in phase by $90^{\circ}$ (one-quarter cycle). These signals have the same period and a fifty-percent duty cycle. The signal which leads in phase changes with the direction of axis motion. An additional signal, called the reference or index, produces one pulse per revolution of the encoder. This signal, which is used when the axis is referenced, will not be modified by the RTEC and is not shown in the Figure. The frequency of the square waves is a function of the number of lines per revolution in the encoder (perhaps 1024) and the rpm of the encoder. Therefore, at rest, the two signals are static and either may be at high or low logic levels. Figure 3a shows the A and B signals with the axis moving in the positive direction (phase A leads phase B). Each cycle of the square waves corresponds to a linear distance of axis movement which depends on the number of lines per revolution in the encoder, the gear ratio between encoder and the ball screw and the ball-screw pitch. To measure position, the encoder-type version of the RTEC must contain circuitry to count the encoder cycles and determine which direction the axis is moving in order to know whether to add or subtract counts. This circuitry will give more position resolution, by a factor of 4 , than the encoder resolution. In compensation mode, to make the machine axis stop sooner by the distance corresponding to one square-wave cycle, the RTEC must insert extra pulses with the correct phase relationship as shown in Figure 3b. Likewise, to make the axis go one cycle further, the RTEC must subtract one pair of pulses as in Figure 3c. Note that the resolution of the correction is the displacement corresponding to one square-wave cycle. This correction resolution is four times coarser than the axis position resolution, which is typically one or two micrometers.

\section{$\underline{\text { Implementation }}$}

The RTEC is a microcomputer-based device, containing one single-chip microcomputer for each axis of the machine tool. Thus, there are typically two, three or more microcomputers in the RTEC used for the error compensation of one machine tool, with each one communicating with the PC.

The initial implementation discussed here is an RTEC device used with a machine tool with encoder-type axis-position feedback signals. Specifically, the first machine tool is a two-axis $25 \mathrm{hp}$ $(18.6 \mathrm{~kW})$ Monarch Metalist turning center (lathe) with a General Electric Mark Century 2000 controller. For position feedback, this machine tool uses linear glass scales with electronic conditioning that produces encoder-type signals. To demonstrate the ability to implement compensation, preliminary tests have been carried out, with a limited prototype of the RTEC, using this machine tool. Though this prototype did not contain a microcomputer, the physical locations of the axes could be modified without intrusion into the MTC and without creating error conditions. 
The following explanation of the RTEC is simplified for clarity. It is assumed for this explanation that only one axis is being controlled by the RTEC. A more detailed explanation will follow below. The implementation of the RTEC is shown in Figure 4. The encoder quadrature position-feedback signals are square-wave signals $A$ and $B$ in Figure 3 displaced in phase by $90^{\circ}$ ) They pass from the machine-tool through the RTEC. An 8-bit microcomputer (for each axis) from the 8051 family, sends similar quadrature signals on to the MTC and communicates with the PC.

There are three possible modes of operation for the RTEC. The first is a transparent mode, and in this mode the RTEC merely passes the quadrature position-feedback signals, unchanged, to the MTC. The transparent mode is required for conventional, uncorrected operation of the machine tool. The second mode is the probing mode and the third mode is the correction mode. In all modes, the encoder signals go to a quadrature decoder within the RTEC. Here, the direction of axis movement is determined from the phase relationship between $A$ and $B$. The signals are decoded into counts, each count having one-quarter the resolution of the quadrature input. Depending upon the direction of axis movement, a counter adds or subtracts these counts, and a continuously updated total is maintained. The number of counts, when multiplied by the resolution of one cycle, gives the absolute position of that axis with respect to the axis reference or home position.

In probing mode, the probe-trip signal holds the trip position at the decoder output until the microcomputer can read the position. The decoder is designed to continue to count the encoder signals to track the machine position. The microcomputer then outputs the trip position to the PC. In the correction mode, the microcomputer does several things. It transfers the quadrature waveform received from the machine-tool feedback device to the MTC. It also continually reads the number of counts being accumulated in the quadrature decoder/counter unit described above. The axis position in counts is periodically sent to the PC/AT computer. The PC will calculate a position correction for each axis based upon the current axes positions and temperature data and send this correction back to the RTEC. The RTEC actively changes the quadrature feedback signal, before passing it on to the MTC, in response to the correction commands.

The RTEC interprets this correction as the number of pulses that must be added to (or withheld from) the actual quadrature signal before it is passed through to the MTC. The MTC has a quadrature decoder/counter also, and when additional pulses are inserted by the RTEC, it will count these and reach its own predetermined destination count (position) that much sooner. By the same reasoning, if some of the axis-encoder pulses are withheld from the MTC, the axis will be kept in motion for a longer distance -- enough to compensate for the distance represented by the withheld cycles. For example, if one encoder cycle represents an axis distance of about 160 microinches (4 micrometers), and an axis move is to be from the 1.000 -inch $(25.400-\mathrm{mm})$ position to the 2.000 -inch $(50.800-\mathrm{mm}$ ) position, then adding six pulses (approximately 0.001 inch) will cause the axis to stop at the 1.999-inch $(50.776-\mathrm{mm})$ position. Withholding six pulses, however, will cause the axis to continue to the 2.001-inch $(50.824-\mathrm{mm})$ position. Note, however, that in either case the MTC will display 2.000 inches $(50.800 \mathrm{~mm})$ as the position. The correction implemented by withholding pulses will work whether the direction of axis movement is negative or positive. However, if a correction is desired which entails adding pulses, then the additional $A$ and $B$ pulses must have the correct phase relationship. 
A more detailed view of the correction process at the RTEC microcomputer level includes the pulse-counting operation, the generation of additional pulses, communication with the PC and potential problems. First, it should be noted that the entire RTEC operation must begin with all axes at the machine tool's home position. Once the machine tool is in this position, the RTEC position counters must be reset.

As mentioned, decoding the incoming A and B signals and maintaining a count, adding or subtracting depending on direction, will be handled by a decoder/counter module. When the counter reaches its maximum count (4095), it goes back to zero (rolls over) and begins again. The microcomputer periodically reads the output of this counter, and must do so often enough so that even during the most rapid axis movement, the count remains unambiguous, even if the counter has rolled over or the direction of axis movement has changed. The total position count that the RTEC sends to the PC is the actual physical location relative to the machine home position of that axis, as seen by the feedback device. At a particular position, this actual count will differ from the MTC internal count, and display, by the amount of the error correction.

Altering the encoder signals to the MTC by adding or withholding pulses must be done in a way that does not cause the MTC to detect an interruption in the proper sequence of signals. Figures $3 \mathrm{a}$ and $3 \mathrm{~d}$ illustrate the correct sequence of rising and falling edges for signals $\mathrm{A}$ and $\mathrm{B}$ in both the negative and positive directions of axis movement. The most straightforward way to implement this correction is to pick a state, when both A and B are low, for example, and only add pulses (or withhold them) during this time (Figures $3 \mathrm{~b}$ and $3 \mathrm{c}$ ). This simplifies the task of the microcomputer during pulse addition because it needs only to generate one $A$ and $B$ sequence for each axis direction. Similarly, it needs only to check for one sequence when withholding pulses. There is no restriction that the width of the added pulse be the same as the width of the pulses before or after it. It is allowable to insert short $A$ and $B$ pulses whether the axis is travelling at $20 \mathrm{in} / \mathrm{min}(500 \mathrm{~mm} / \mathrm{min})$ or $200 \mathrm{in} / \mathrm{min}(5000 \mathrm{~mm} / \mathrm{min})$. This simplifies the work of the microcomputer because it has to generate pulses of only one width.

The most important information communicated between the PC and the RTEC is the axes positions (sent from the RTEC to the PC) and the axes corrections requested of the RTEC by the PC. The digital word which represents the axis position (in counts) must be large enough to depict the number of counts in that axis. The longest axis in this turning center ( $\mathrm{Z}$-axis) is approximately 40 inches ( 1 meter), and since each count (i.e., after quadrature decoding) represents 40 microinches ( 1 micrometer), the axis-position word must go up to a million. If 20 bits are used, the RTEC can keep track of up to $1,048,575$ position counts. For the axis correction, it is expected that a \pm 20 milliinch $( \pm 0.51$ millimeter) correction range will be adequate. Since the corrections will be made with respect to an encoder cycle, not counts, and each cycle on this turning center represents approximately 160 microinches ( 4 micrometers), an 8-bit word ( \pm 127 correction counts) will suffice.

Communication between the RTEC and the PC takes place through a parallel digital input/output card in the PC. The RTEC will tell the PC how many correction units it has actually implemented (for comparison with the number requested). Additional bits are used for control (handshaking) of the information transfer. As mentioned above, the RTEC will control the corrections for two or more machine tool axes. Each axis, with its dedicated microcomputer, will have its own set of communication lines to the PC. 
A limitation of the encoder version of the RTEC for error compensation is the resolution of its corrections. An MTC receives quadrature signals and decodes them internally with a resolution, usually four times finer than the incoming cycles. Since the RTEC can only make corrections by adding or withholding whole cycles, the correction resolution is four times coarser than the position resolution.

\section{Fast Probing}

The requirement that the part location be known for fast probing is inherently satisfied in part inspection on a CNC machine tool. The part has just been cut and the location is known within the accuracy of machining. A program to move the probe can be written, in advance, from the same drawing or data used to generate the machining part program. The number of data points and the expected values are known within the machining error. The MTC is unaware of the probing taking place. The parallel data link from the RTEC to the PC bypasses the slow DNC data link to the MTC. There are, however, many considerations to achieve successful fast probing.

Probes are specified for relatively slow feed rates of $18 \mathrm{in} / \mathrm{min}(450 \mathrm{~mm} / \mathrm{min})$ and, up until now, used at even slower feed rates (about $5 \mathrm{in} / \mathrm{min}[127 \mathrm{~mm} / \mathrm{min}]$ ). The probe and its electronic interface has significant, but repeatable, time delay from probe trip to electrical signal output. At $100 \mathrm{in} / \mathrm{min}$ $(2540 \mathrm{~mm} / \mathrm{min})$, the machine moves one micrometer (the position resolution) in 24 microseconds; the probe delay variability is an order of magnitude smaller. For precision probing, the effect of the delay, the probe tip diameter, and the effect of probe trip-force and pretravel must all be carefully calibrated at each velocity and direction of approach to be used. The probe pretravel and trip-force have a three-lobed characteristic in the radial direction and probes have higher trip-force in the axial direction.

For optimum repeatability in part inspection, the probe must trip during the constant velocity (programmed feed rate) part of each point-to-point move. For locating part blanks during set up, this is not required. Usually, machines accelerate at approximately a constant rate until the programmed velocity is approached and maintain constant velocity until the end point approaches (figure 5). The drive motors then brake to a stop. The acceleration and deceleration times (and the corresponding distances) are a function of the specific axis drive system. The actual machine position lags the MTC programmed position due to following error which is a function of servo-loop gain. For this turning center, the following error is about 70 milliinches $(1.78 \mathrm{~mm}$ ) at $100 \mathrm{in} / \mathrm{min}$ $(2540 \mathrm{~mm} / \mathrm{min})$ feed rate. The starting point and end point of the programmed probe motion must be chosen to ensure tripping during the constant velocity part of the move. To allow time for acceleration to $100 \mathrm{in} / \mathrm{min}(2540 \mathrm{~mm} / \mathrm{min})$ the probe must start about $0.2 \mathrm{in}$. $(5.1 \mathrm{~mm})$ from the part and move to $0.1 \mathrm{in} .(2.5 \mathrm{~mm}$ ) beyond the part edge to allow time to decelerate (which must be within the probe overtravel limit). This results in 0.12 in. ( $3 \mathrm{~mm}$ ) of constant velocity travel. A variation in part position (trip point) of \pm 0.04 in. $( \pm 1 \mathrm{~mm})$ could be tolerated. Since the probe trip signal holds the true position count from the feed-back device, the servo lag does not cause a dimensional error. 
We have demonstrated the effectiveness of fast probing on our turning center. Six points on a part were probed in 11 seconds--one-third the time used by typical slow feed rates. Repeated probing of the same point on the part shows a two sigma repeatability of one micrometer which happens to be the position feedback resolution and the probe repeatability specification. The probe retract is performed at machine traverse rate which is $275 \mathrm{in} / \mathrm{min}(6980 \mathrm{~mm} / \mathrm{min})$. The same point can be probed at the rate of two per second. Considerable time is used to move between probing locations. Since our probing data is in the PC, coordinate measuring machine (CMM) type data analysis can be performed while machining continues.

\section{$\underline{\text { Summary }}$}

A device (the RTEC) has been constructed which facilitates fast probing and will soon allow realtime compensation for systematic errors in machine tools. The device is inserted between the position feedback element (in this case an encoder-type output device) and the machine-tool controller so that the RTEC is nonintrusive to the controller. The RTEC interfaces with a PC/AT compatible computer that records probing data and calculates corrections. The RTEC was initially installed on a two-axis turning center. Its design is not specific to a particular MTC and will not require significant hardware or software modifications for different controllers with encoder-type feedback. In practice, however, the type of position feedback and resolution needs to be known, as well as the encoder/MTC interface circuitry.

The feasibility of performing on-machine inspection with fast probing at one data point per second and a repeatability of one micrometer has been demonstrated. The MTC, which is unaware of the probing taking place, merely executes a part program which moves the probe into and out of the part at the desired probing locations. The standoff and over-travel distances for the probing moves must be chosen to ensure that the probe always trips at constant velocity. Inspection of the part before the final cut, or after completion can be performed without wasting machining time.

The previous implementation of error compensation on a turning center yielded up to a 20-times improvement in accuracy of diameter, length and straightness. It is difficult to predict the improvement to be gained with the RTEC on this turning center because it has not yet been characterized with laser measurements. These measurements must be performed over the operational temperature range, to determine the magnitude of the errors. We may not realize as large an accuracy improvement as in the past because this

turning center uses glass scales instead of resolvers for position feedback, so there are no ball-screw errors. The final proof of the RTEC and the error compensation model is to test the results by cutting (from a cold start) several test parts, then checking the accuracy of the parts. This will check the accuracy of both of the error-compensation components--the thermal error compensation (as the lathe warms up) and the geometric error compensation.

In conclusion, this fast probing and error compensation system will provide significant benefits. More complete, on-machine, part inspection can be performed without wasting machining time. From previous experience on a turning center, we believe an accuracy improvement of up to an order of magnitude can be achieved over the operating temperature range. Finally, the RTEC is applicable to a wide range of machine tools because it is independent of the MTC. 


\section{Acknowledgments}

We would like to thank Leo Sommerville and David Pickle of Renishaw Inc. who suggested that a black-box could be built to facilitate fast probing and who provided the probes used. Also, thanks to Ralph Prescott of Monarch Sidney for providing a special holder for probes.

\section{$\underline{\text { References }}$}

1. Technology of Machine Tools / Machine Tool Accuracy, Vol. 5, ed. Robert J. Hocken, Pub. Lawrence Livermore National Laboratory, Livermore, Calif., 1980.

2. Donmez, M. A., Blomquist, D. S., Hocken, R. J., Liu, C. R., and Barash, M. M., "A General Methodology for Machine Tool Accuracy Enhancement by Error Compensation," Prec. Eng. 4: $187,1986$.

3. Donmez, M. A., Liu, C. R., and Barash, M. M., "A Generalized Mathematical Model for Machine-Tool Errors," Modeling, Sensing, and Control of Manufacturing Processes, PEDVol.23/DSC-Vol.4, Book No. H00370, Eds. K. Srinivasan, D.L.E. Hardt, and R. Komanduri, American Society of Mechanical Engineers, New York, 1987.

4. Miller, Paul C., "Automatic Setup for Machining Centers," Tooling and Production, May, 1987.

5. Donmez, M. A, Lee, K L., Liu, C. R., and Barash, M. M., "A Real-Time Error Compensation System for a Computerized Numerical Control Turning Center," Proc. 1986 IEEE Int. Conf. on Robotics and Automation, April 7-10, 1986.

6. Boyes, Geoffrey S., Synchro and Resolver Conversion, Memory Devices Ltd., Surrey, U.K. 1980 


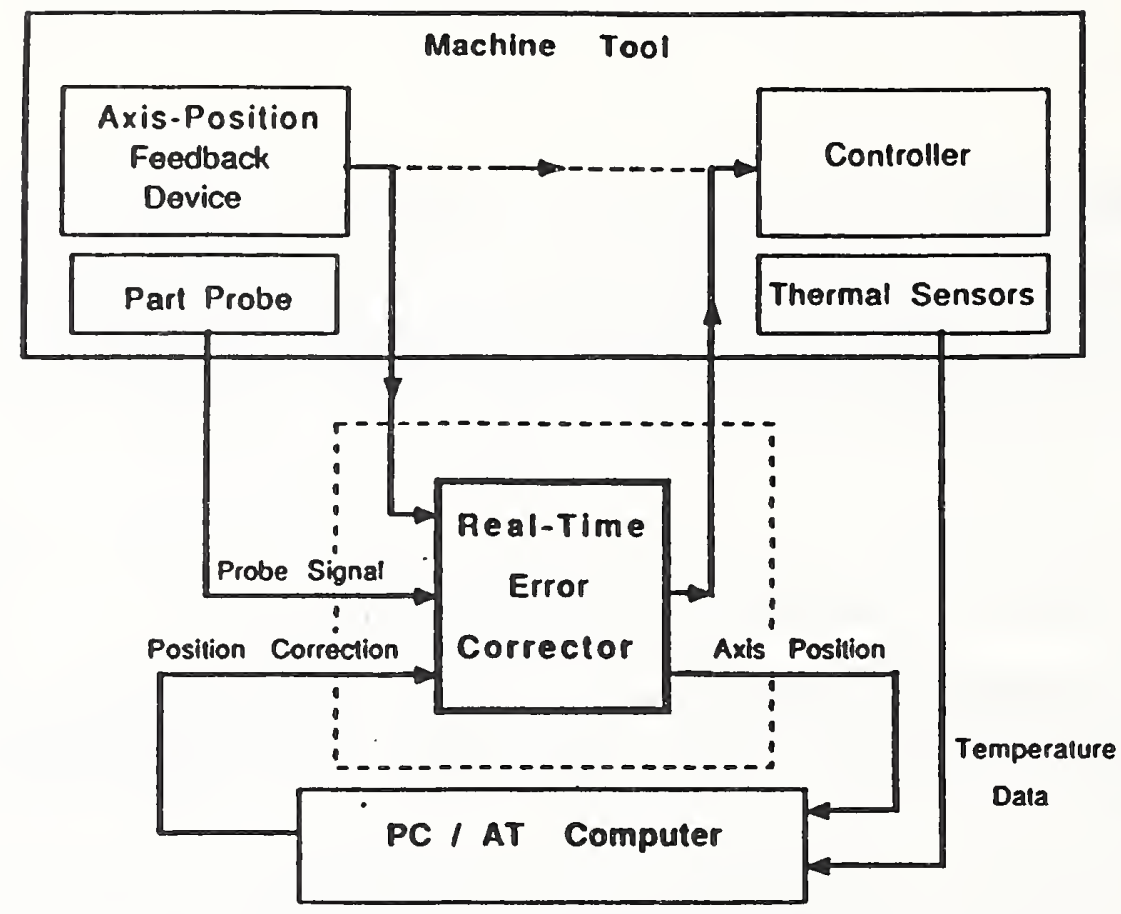

Figure 1 System Overview

Reference

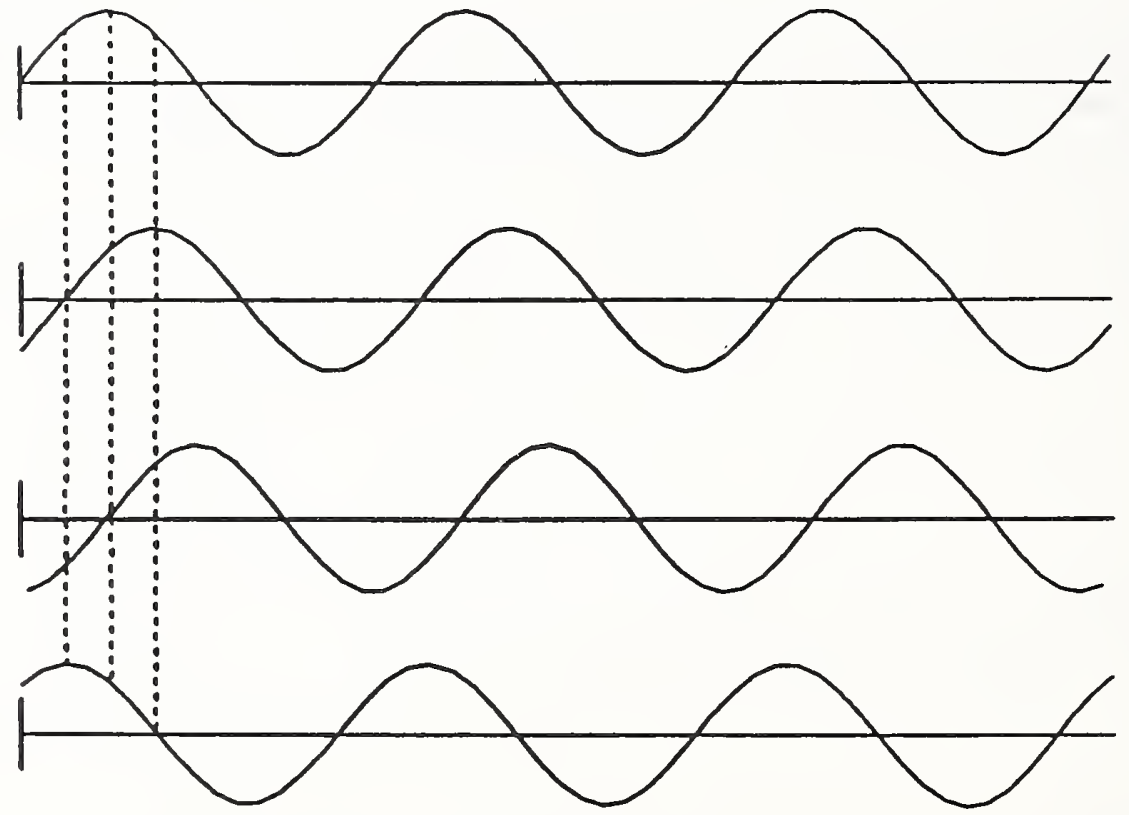

a. Output with axis stopped.

b. Output after axis has moved 1/8-resolver-rotation positive.

c. Output atter axis has moved 3/8-resolver-rotation negative from (b) or 5/8-resolver-rotation more positive from (b).

Figure 2 Resolver Signals 

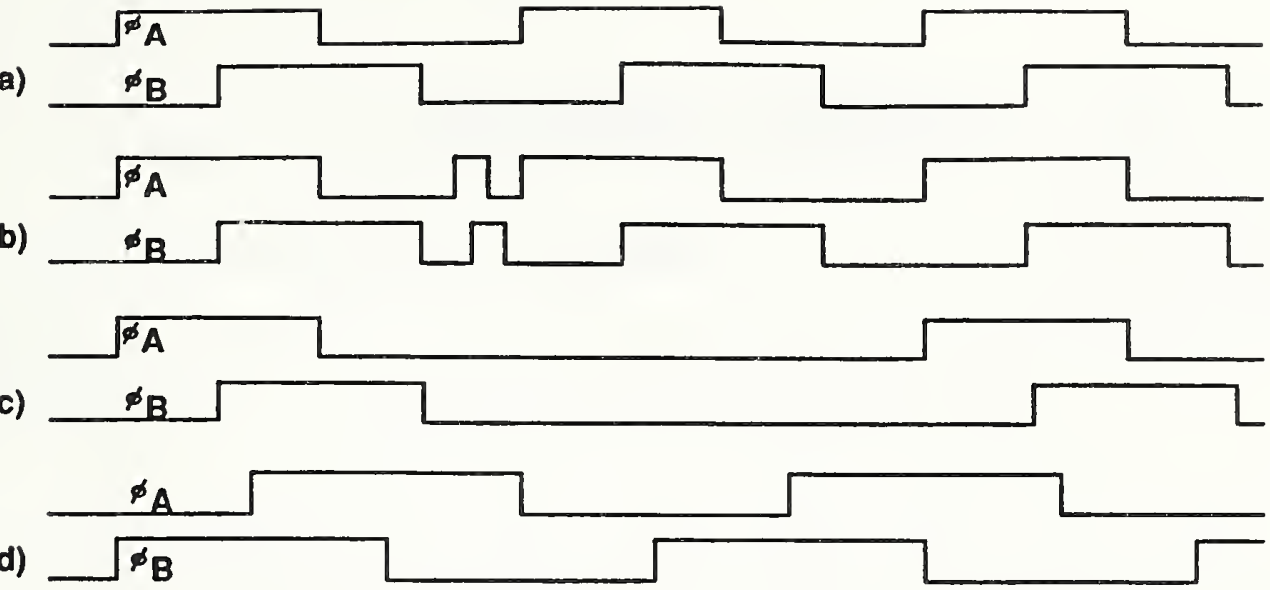

a.Axis moving in positive direction. b. One pulse added.

c. One pulse withheld. d.Axis moving in negative direction at a slower rate.

Figure 3 Encoder Signals

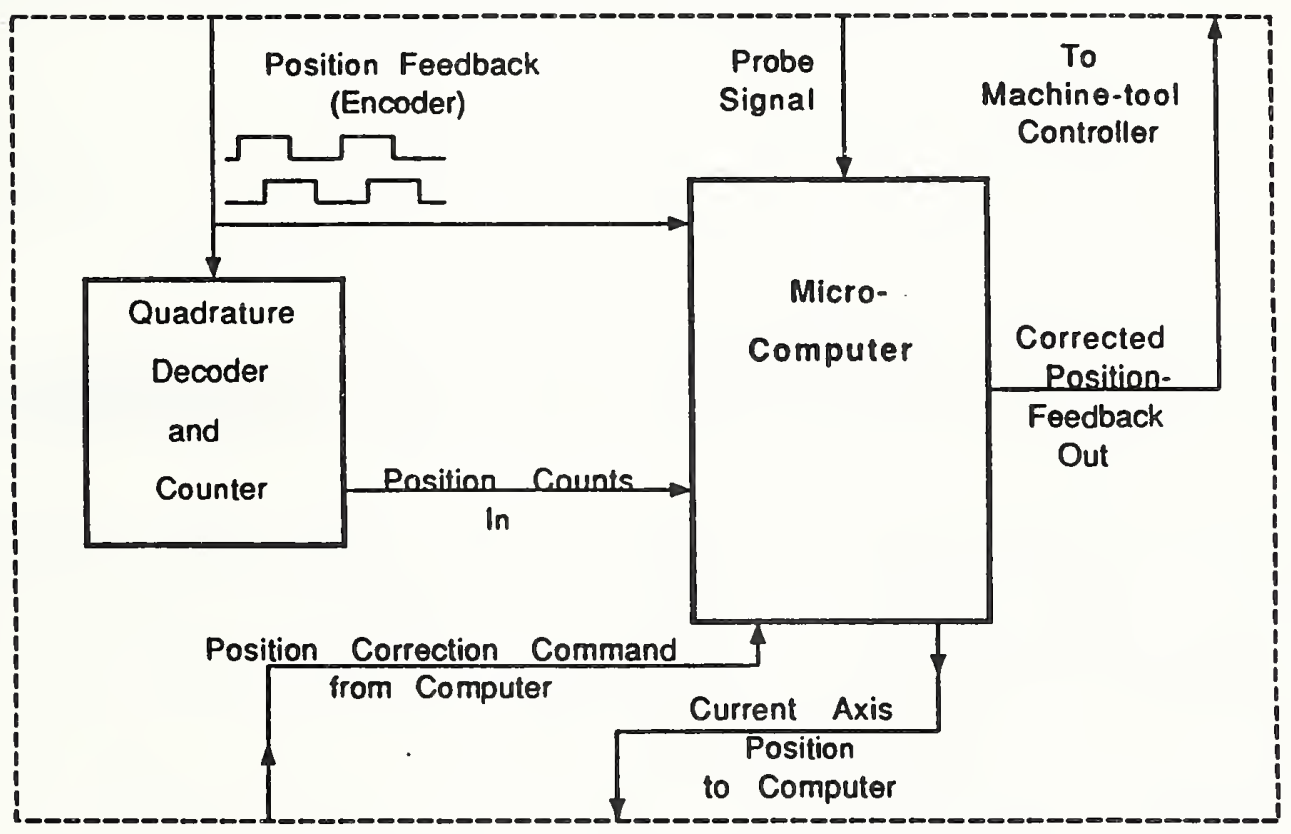

Figure 4 Real-Time Error Corrector

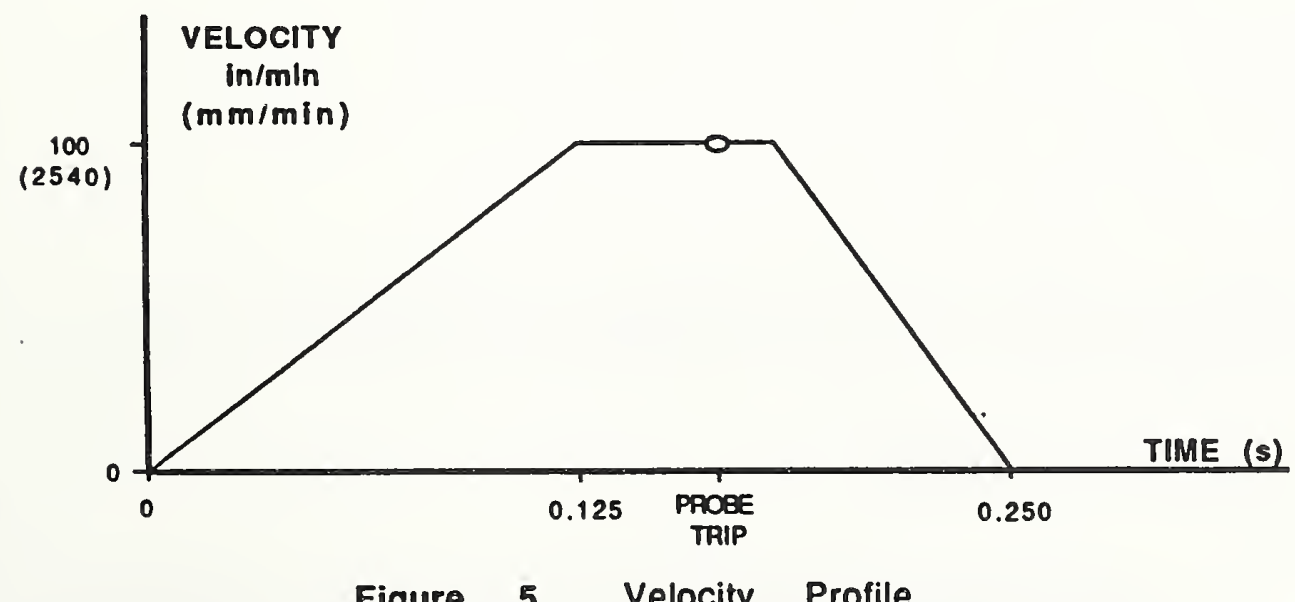

Figure 5 Velocity Profile 


\begin{tabular}{|c|c|c|}
\hline \multirow[t]{4}{*}{$\begin{array}{l}\text { NIST-114A } \\
\text { (REV. 3-90) }\end{array}$} & \multirow[t]{2}{*}{$\begin{array}{r}\text { U.S. DEPARTMENT OF COMMERCE } \\
\text { NATIONAL INSTITUTE OF STANDARDS AND TECHNOLOGY }\end{array}$} & $\begin{array}{l}\text { 1. PUBLCATION OR REPORT NUMBER } \\
\text { NISTIR } 4447\end{array}$ \\
\hline & & 2. PERFORMING ORGANIZATION REPORT NUMBER \\
\hline & \multirow[t]{2}{*}{ BIBLIOGRAPHIC DATA SHEET } & 3. PUBUCATION DATE \\
\hline & & October, 1990 \\
\hline
\end{tabular}

4. TITLE AND SUBTITLE

Implementing Fast Part Probing and Error Compensation

on Machine Tools

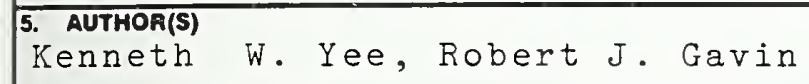

6. PERFORMING ORQANIZATION (IF JOINT OR OTHER THAN NIST, SEE INSTRUCTIONS)

U.S. DEPARTMENT OF COMMERCE

MATIONAL INSTITUTE OF STANDARDS AND TECHNOLOQY

GAITHERSBURG, MD 20890

8. TYPE OF REPORT AND PERIOD COVEAED

9. SPONSORING ORGAMIZATION NAME AMD COMPLETE ADDAESS (STREET, CITY, STATE, ZIP)

10. SUPPLEMENTARY NOTES

11. ABSTRACT (A 20O-WORD OR LESS FACTUAL SUMMARY OF MOST SIQNIFICANT INFORMATION. IF DOCUMENT INCLUDES A SIQNIFICANT BIBLOGRAPHY OR UTERATURE SURVEY, MENTION IT HERE.)

A device has been constructed to facilitate fast probing and real-time error compensation on CNC machine tools without intrusion into the machinetool controller. This device, interfaced to a PC computer, is inserted between the position feedback elements and the controller. Fast probing using a touch-trigger probe is achieved by providing high-speed access by the PC to the probe-trip positions. The device performs error-compensation by modifying the position-feedback signals going to the controller. Pulses from the "encoder" are added or subtracted to alter the position count in the controller. The correction values are calculated by the PC using a model previously developed at NIST for predicting, in real-time, machine-tool geometric and thermal errors.

12. KEY WOADS (6 TO 12 ENTRIES; ALPHABETICAL ORDEA; CAPITALZE ONLY PROPER NAMES; AND SEPARATE KEY WORDS BY SEMICOLONS)

encoder; error compensation; in-process; machine tool;manufacturing; probing; real-time

13. AVALLABIUTY

\begin{tabular}{|l}
\hline$X$ \\
\hline \\
\hline
\end{tabular}

UNLMITED

FOR OfFICIAL DISTRIBUtION. DO NOT RELEASE TO MATIONAL TEChNICAL INFORMATION SERVICE (NTIS).

ORDER FROM SUPEAINTENDENT OF DOCUMENTS, U.S. QOVERNMENT PRINTING OFFICE, WASHINOTON, DC 20402

ORDER FROM HATIONAL TECHNICAL INFORMATION SERVICE (NTIS), SPRINGFIED, VA 22161.

14. NUMBER OF PRINTED PAGES

14

15. PRICE

$\mathrm{A} 02$ 




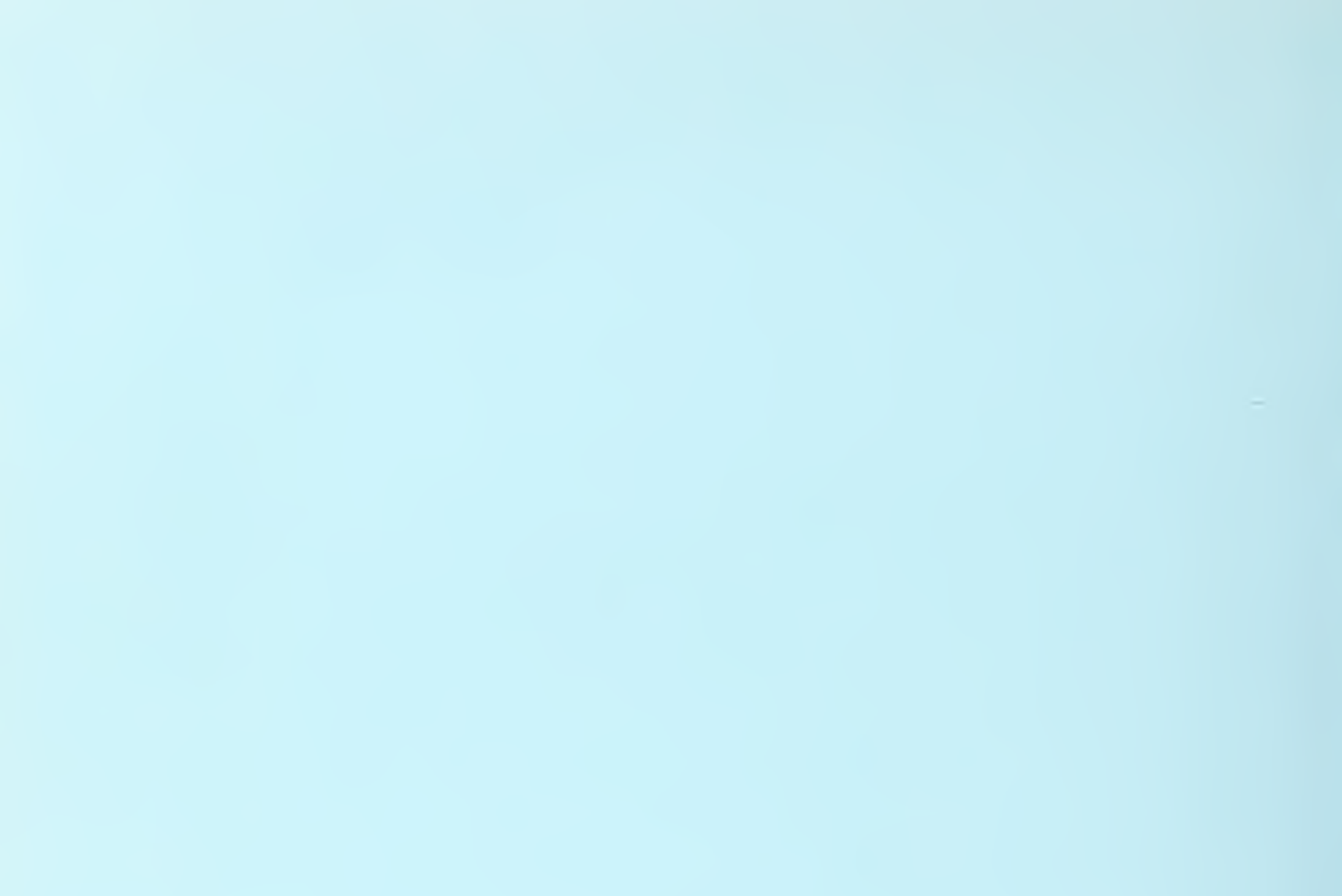

